\title{
L'HOMME L'Homme
}

Revue française d'anthropologie

\section{5-216 | 2015}

\section{Connaît-on la chanson?}

\section{Cette chanson est pour vous, Madame...}

Les années chanson française de Django Reinhardt, 1933-1936

"Cette chanson est pour vous, Madame...". Django Reinhardt's French Popular

Music Years, 1933-1936

\section{Patrick Williams}

\section{OpenEdition \\ Journals}

Édition électronique

URL : http://journals.openedition.org/lhomme/23909

DOI : 10.4000//homme.23909

ISSN : 1953-8103

Éditeur

Éditions de l'EHESS

Édition imprimée

Date de publication : 13 novembre 2015

Pagination : 103-126

ISSN : 0439-4216

Référence électronique

Patrick Williams, "Cette chanson est pour vous, Madame... », L'Homme [En ligne], 215-216 | 2015, mis en ligne le 12 novembre 2017, consulté le 20 avril 2019. URL : http://journals.openedition.org/ Ihomme/23909; DOI : 10.4000/lhomme.23909 


\section{Cette chanson est pour vous, Madame... \\ Les années chanson française de Django Reinhardt, 1933-1936}

Patrick Williams

$\mathrm{D}$

JANGO REINHARDT (1910-1953) a découvert la musique dans l'orchestre de son père. La caravane manouche allait de ville en ville, donnant des représentations où se mêlaient musique, danse, numéros d'acrobates et de funambules, tours de magie, saynètes de théâtre dans des salles d'auberge, sur des places publiques, sur des tréteaux dressés à l'occasion d'une fête de village... En même temps, l'enfant s'essayait à la mandoline, à la guitare, au violon et apprenait à connaître les routes d'Europe (et au-delà) : il est né à la Mare au Flachs ("Mare aux Corbeaux »), un lieu-dit près du bourg de Liberchies, dans la province du Hainaut, en Belgique, et la seule photographie que nous possédions de cet orchestre a été prise à Alger, en 1915, Django avait cinq ans. Elle nous montre les membres de la famille posant avec leurs instruments : des guitares, des violons, une contrebasse (et dans un coin de la scène, le père assis devant un piano invisible). On se plaît alors à imaginer une trajectoire qui aurait conduit Django des jours obscurs de la musique sur les routes - le chapeau ou la soucoupe que l'on passe parmi le public à la fin de chaque morceau - aux lumières de la renommée avec les premiers succès du Quintette du Hot Club de France: d'un orchestre à cordes à un autre orchestre à cordes.

Une version de la première partie de ce texte a été publiée, en janvier 2010, dans le numéro hors-série Django 1910-2010 de Guitarist Acoustic Magazine, à l'occasion du centenaire du guitariste ("Cette Chanson est pour vous: étude sur Django et les chanteurs/chanteuses de variété française entre 1933 et 1936 », pp. 8-13). Ce texte a également servi de support à l'exposé présenté au séminaire "Chant, poésie et mythe populaires" du duo Daniel Fabre-Jean Jamin, à l'École des hautes études en sciences sociales, le 13 avril 2012. 
Mais les choses ne se sont pas passées comme cela. Quand la roulotte s'arrête aux portes de Paris, dans ce territoire interlope qu'on appelle la zone, c'est aux côtés des caïds de l'accordéon - le musette, genre nouveau, triomphe - que le jeune virtuose se forge une réputation; il joue alors du banjo à six cordes, instrument moins subtil que la guitare mais plus puissant. Son ascension est stoppée en novembre 1928 par les terribles brûlures qu'il subit lors de l'incendie de sa roulotte. Il faudra plus de trois ans de soins et de rééducation pour qu'il retrouve toutes ses facultés et qu'il rejoue de la guitare. Comme avant? On ne le saura jamais : l'annulaire et l'auriculaire de la main gauche ont perdu toute mobilité. Durant les mois partagés entre les séjours à l'hôpital et les retours à la roulotte de sa mère, Django a inventé pour lui-même une nouvelle technique, adaptée à son infirmité. Quand, en 1933, il réapparaît dans les studios d'enregistrement, il accompagne chanteuses réalistes et chanteurs de charme, célèbres ou pas, talentueux ou pas, sensibles aux échos du "jazz nègre ", comme on disait alors, ou pas. C'est en cette compagnie qu'il passe de la musique de danse populaire à ce que nous appellerions aujourd'hui la variété; qu'il bascule aussi dans le métier. Et, jusqu'à la fin de 1936, il va alterner les prestations avec ces vocalistes, les premières gravures et les premiers concerts avec le Quintette à cordes, les collaborations comme sideman dans les toutes nouvelles formations de jazz composées de musiciens français ("Michel Warlop et son Orchestre ») ou dans les ensembles qui creusent un sillon entre jazz et variété ( Patrick et son Orchestre de danse» - "Patrick» est le tromboniste André Paquinet). Le jazz est dans l'air; chacune de ces formations en propose une déclinaison qui affirme son originalité par rapport aux modèles américains. On y aperçoit ceux qui deviendront les premières figures du jazz à la française, s'appariant dans différentes configurations au gré des séances d'enregistrement: Django Reinhardt et Stéphane Grappelli bien sûr (ce dernier aussi souvent pianiste que violoniste), et Michel Warlop, André Ékyan, Alix Combelle, Noël Chiboust, Alex Renard, Pierre Allier... Mais des Américains sont présents à Paris, résidents ou de passage, qui s'invitent à ces réjouissances: Coleman Hawkins, Fletcher Allen, Arthur Briggs, Big Boy Goudie, Garnett Clark, Bill Coleman...

\section{Comme un aérolite}

C’est sur ces quatre années au cours desquelles Django Reinhardt a accompagné les interprètes de quelque soixante chansons (voir la liste de ces chansons en fin d'article), que j'ai choisi de m'arrêter. Il ne me paraît pas possible en effet de tenir ces pièces gravées en compagnie d'interprètes 
certainement représentatifs de la chanson française en cette première moitié des années 1930, même si aujourd'hui certaines d'entre elles paraissent bien désuètes, comme des opportunités purement alimentaires qui ne compteraient pour rien dans l'évolution musicale de Django. Au contraire: quelques-unes constituent des jalons dans l'éclosion du "guitariste suprême" (comme le qualifiait Philippe Carles) et l'on s'aperçoit que cette étape de son parcours l'a aidé à mûrir. Ce qui frappe d'abord à l'audition des soixante titres de ce corpus, c'est qu'à chaque fois, qu'il s'impose en solo sur le devant de la scène, qu'il introduise d'un chapelet de notes ou de deux ou trois accords enchaînés, qu'il brode un lointain contre-chant derrière la voix, Django est là tout entier. Le son, la découpe des phrases, la construction de la séquence : aucun doute, nous sommes en face de "l'astre sans parenté connue dans la musique populaire de l'époque». Du coup, l'auditeur ne se demande plus «qu'est-ce que Django Reinhardt est venu faire dans cette galère? ", mais "comment la tragédienne aux semelles de plomb ou le charmeur chevrotant ont-ils pu accepter la présence de ce guitariste qui leur vole leur chanson ? ". Car une fois que l'on s'est laissé prendre à suivre la guitare, on n'entend plus qu'elle.

Aussi ne pourrait-on commenter qu'un seul de ces titres pour rendre compte de ce qui se passe dans chacun d'eux : le hold-up que Django réussit à tous les coups. Deux notes et ça y est, il a tout raflé! Celles, par exemple, de l'introduction à Je sais que vous êtes jolie, avec Jean Sablon, le 11 avril 1934. Je dis "introduction" mais "apparition" serait plus juste: Django se présente, il se déploie. En fait, il chante : sa guitare est une guitare et une chanteuse en même temps. Deux notes, une pause: il prend son temps... Voilà sa force: prendre le temps. Puis la chanson se déroule, plutôt réussie, alternant séquences hors-tempo ("Vraiment Monsieur je voudrais pourtant savoir / Pour quel motif vous me suivez tous les soirs / Chaque fois qu'il faut que je sorte / Je vous retrouve à ma porte »), reprise syncopée, refrain à l'allant nonchalant ("Je sais - que vous êtes jolie / Que vos grands yeux pleins de douceur / Ont charmé - tout mon cœur / Et que c'est pour la vie»). L'entente entre la guitare magicienne, la clarinette boisée d'André Ékyan, le piano mélodieux d'Alec Siniavine et le chanteur fait merveille, il y a bien là une sorte de miracle : l'alliance d'une force à laquelle rien ne semble pouvoir résister - et ce d'autant plus qu'elle s'impose en douceur - et d'une pertinence absolue rien dans ce que propose la guitare n'est en trop -, ces trois minutes offrent une image de la perfection.

Tous les interprètes ne réagissent pas de la même façon à cet accompagnement miraculeux. Jean Sablon, chanteur de charme et fantaisiste tout à la fois, est demandeur. Sur la scène des music-halls, il met les musiciens 
à ses côtés alors que l'habitude était de les cantonner dans la fosse d'orchestre ; il se bat avec les dirigeants de la puissante Columbia, qui l'a "signé ", pour imposer un solo (huit mesures, seize mesures) de Django dans chacune des chansons qu'il enregistre. Dès les premières collaborations (Le Même coup; Je suis Sex-Appeal - "Je ne suis pas beau gosse / Mais je suis Sex-Appeal / Qui m’approche s'électrise / Je suis comme une pile!-; Le jour où je te vis), la complicité, l'humour et le bonheur qu'ils trouvent à jouer ensemble sont manifestes. D'autres, comme Pierre Lord, totalement chanteur de charme lui, semblent un moment désarçonnés mais s'en remettent: comment, dans Cocktails pour deux, fait-il pour attaquer "Que de séduisants rendez-vous..." après l'enchaînement d'accords que vient de proposer Django, véritable premier jet de ce qui deviendra son introduction célébrissime de When Day Is Done? On n'en sait rien, mais il attaque. Sur Fumée aux yeux (le Smoke Gets In Your Eyes de Jerome Kern), une complicité s'établit entre Django et lui pour une interprétation gentiment humoristique ("Ceux qui blaguent l'amour / Me disent toujours / Que pour des amants / Rien n'est plus troublant / Que l'aveuglement...»), et hop, petite ponctuation maligne de la guitare! Aimé Simon-Girard est à la peine. Léon Monosson, malgré ses grands airs et ses effets appuyés, déraille assez vite; du coup, que les quelques mesures en solo que s'accorde le guitariste dans Deux Cigarettes dans l'ombre apparaissent comme un cadeau incongru. Les plus étonnants sont ceux ou celles qui ne s'aperçoivent de rien ou semblent ne s'apercevoir de rien et qui, après l'intervention de Django, poursuivent ou reprennent comme si effectivement rien ne s'était passé! Pourtant, le coup de vent qui vient de souffler sur leur chanson a transformé tout le paysage. Germaine Sablon apprécie peut-être ces brises fécondes (dans La Chanson du large, c'est un calme cyclone qu'elle doit affronter), mais elle ne se départ jamais du sérieux avec lequel elle déclame ses chansons à texte.

Nombre de ces pièces apparaissent aujourd'hui datées et n'auraient d'intérêt qu'historique n'étaient les interventions du guitariste. Parfois celui-ci semble, à travers un arpège, un coup de poignet, prendre plaisir à souligner le caractère inapproprié du cadre dans lequel il se trouve placé, ainsi dans les drames réalistes que chante Nane Cholet (Si javais été, Ainsi soit-il). Parfois, il se fait presque invisible, égrenant en guirlande un commentaire si discret derrière la chanteuse ou le chanteur (Un jour sur la mer, adaptation française de I Cover the Waterfront, Deux Cigarettes dans l'ombre, Ici l'on pêche, avec Germaine Sablon; Fièvre - qui n'est pas l'adaptation française du célèbre Fever, mais d'un autre standard bien connu, Moonglow - avec Nane Cholet; Pas sur la bouche, avec Jean Sablon; Petit homme, c'est l'heure de faire dodo, avec Le Petit Mirsha) qu'il 
faut tendre l'oreille pour l'apprécier. Parfois, il se contente de poser la patte sur l'interprétation à venir, en jetant en introduction quelques bribes mélodiques (La Dernière bergère, avec Jean Sablon; Un amour comme le nôtre où le "premier crooner français " est rejoint par sa sœur pour un duo ; Mirage, avec Yvonne Louis, qui est Chasing Shadows dont le Quintette a donné en septembre 1935 une interprétation mémorable) ou quelques accords qui en concentrent la substance harmonique (Simplement, avec Pierre Lord: déjà l'ombre de When Day Is Done se profile; Points roses, avec Nitta Rette). Ces ébauches font entendre qu'au-delà de celui que la chanson va installer, un autre univers existe, ainsi des cinq secondes qui précèdent l'entrée de Germaine Sablon sur Je voudrais vivre: on rêve de suspendre le temps et de se les repasser des centaines et des centaines de fois. Et puis, il y a ces improvisations qui tombent comme un aérolite au milieu d'une chanson, la plus terne (Mon cour reste auprès de toi, avec Nitta Rette : le violon et le piano donnent une introduction légère et swinguante, la vedette attaque "Mon amour pour la dernière fois..." et déjà quelle invention dans les ponctuations de la guitare derrière les gazouillis de la chanteuse! puis soudain, entre deux couplets, l'accident: un solo plein de sursauts, de nœuds et de secousses, à la construction à la fois déroutante et implacable) ou la plus gracieuse (Un baiser, avec Jean Sablon : la guitare introduit brièvement puis le chanteur déroule sur un rythme syncopé tandis que Django et Garland Wilson au piano brodent autour de la ligne mélodique, "... un baiser / D’un taudis dans une cour / Fait un petit nid d'amour ", et à ces mots, la guitare déclenche un extraordinaire solo de funambule, elle détaille la ligne mélodique du refrain pour en faire une illumination). Daniel Nevers en avait repéré un aussi, de ces aérolites, dans La Chanson du large, avec Germaine Sablon; je cite son commentaire :

"À propos de solo, on peut en entendre un, très - trop - bref, mais terriblement impressionnant par l'ineffable magie qu'il distille en quelques mesures, dans La Chanson du large. Si Django n'avait enregistré que ce seul disque, on saurait quand même quel immense musicien il fut $"{ }^{1}$.

Tout aussi saisissant est l'effet que produit le solo de Deux Cigarettes dans l'ombre avec le pénible Léon Monosson. Aucune de ces interventions qui bouleversent l'ordre établi jusque-là par le déroulement de la chanson ne peut être entendue comme déplacée. Au contraire, elles se révèlent toutes excessivement pertinentes. Voici Django face à sa vérité : il est le musicien

1. Cf. le livret du coffret de l'Intégrale Django Reinhardt, 1. Présentation stomp (1928-1934), Vincennes, Frémeaux \& Associés, 1996, non paginé. 
qui se détache des autres par son excès de pertinence - et il lui faudra faire avec ça dans l'univers quotidien, ordinaire que représente si bien le répertoire de ces chanteurs et chanteuses des années 1930.

Jean Sablon, nous l'avons dit, se distingue des autres interprètes. Au-delà de son goût proclamé pour la musique syncopée venue d'Amérique, il a compris quelle était la véritable dimension de ce guitariste que tout le milieu parisien du spectacle trouvait "étrange ». Et certains enregistrements, à la fois par leur atmosphère chaleureuse et leur qualité musicale, nous font bien sentir la connivence qui l'unit au petit groupe de musiciens qu'il a rassemblé autour de lui : Django, son frère Joseph Reinhardt dit Nin-Nin, Stéphane Grappelli, Louis Vola (c'est bien, à une guitare rythmique près, le Quintette du Hot Club de France), André Ékyan et les pianistes Michel Emer, Alec Siniavine et Émil Stern. Alors, Jean Sablon, chanteur du Quintette? Il mériterait ce titre, en tout cas davantage que l'insignifiant Bert Marshall (aussitôt apparu, aussitôt oublié) et l'horripilant Jerry Mengo convoqué pour remplir ce rôle lors d'une séance Ultraphone, le 6 mars $1935^{2}$; celui-ci, mièvre, sirupeux, réussit à faire ce que Jean Sablon évite toujours avec grâce : enliser l'envol de Django et Stéphane - dans Sweet Sue tout de même, la guitare l'expédie par-dessus bord. Il n'empêche, avec Jean Sablon aussi, Django se montre impitoyable. Il suffit de comparer les deux versions, enregistrées en 1935 à moins de deux mois d'intervalle, de Cette chanson est pour vous, Madame. La première, une belle réussite, illustre cette proposition que Jean Sablon pourrait être considéré comme le chanteur du Quintette, Stéphane et Django s'y partageant le chorus instrumental et commentant avec élégance les phrases de l'interprète que Je tire ma révérence et Vous qui passez sans me voir rendront célèbre. La seconde contient l'intervention du guitariste la plus étonnante de cette série. Après l'introduction où, tout de suite, magnifique, la voix de Django s'est posée, Jean Sablon déploie la mélodie en passant savamment les intervalles, porté par la respiration des guitares. Rien n'annonce le plus beau des hold-up jamais réussi par les deux frères (la complicité de Nin-Nin est là certainement précieuse). Le chanteur termine à peine son deuxième couplet que Django tout seul lance une ébouriffante improvisation tandis que Nin-Nin transmue le tempo en une cadence qui rappelle celle du Bolero de Ravel. Django enchaîne les motifs comme un magicien qui déroulerait une échelle de corde dans le vide au fur et à mesure de son ascension. La découpe imprévisible des phrases s'impose avec un air d'évidence. Puis il ramasse le tout

2. Nous ne les avons pas fait figurer dans la liste en fin d'article, recensant les chanteurs et chanteuses accompagnés par Django : tous deux chantent en anglais des succès américains. 


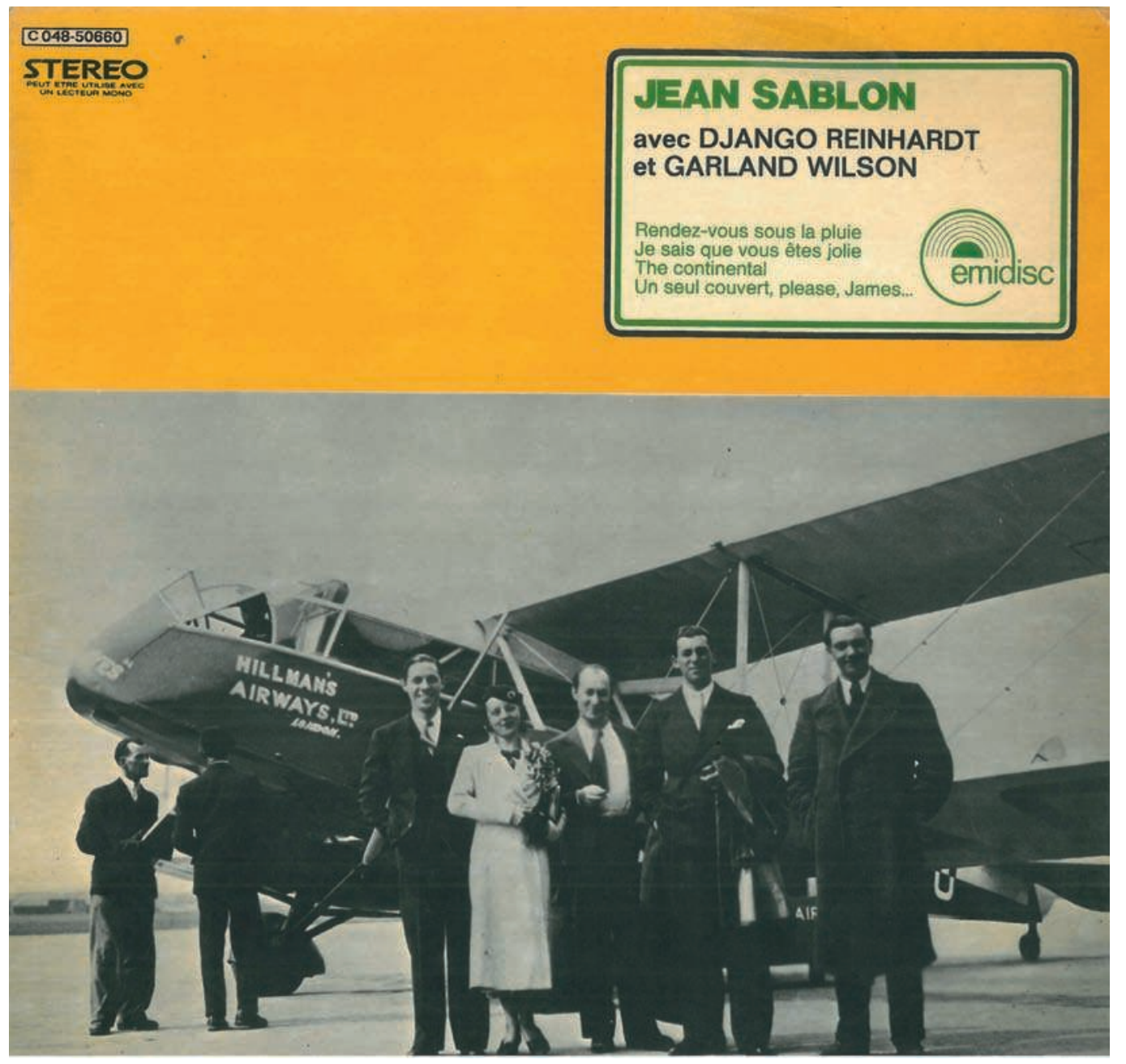

Pochette d'un disque 33 tours, reprise d'enregistrements des années 1933 à 1936, à Paris et à Londres $30 \mathrm{~cm}$, Emidisc Pathé Marconi, s.d. (coll. part.)

De g. à dr. : Jean Sablon, Peddy Nils, Alec Siniavine, André Ekyan, Django Reinhardt [cliché 1934]

d'un large coup de poignet qui reprend la cadence ravelienne. Jean Sablon, en revenant, a bien l'air de s'être rendu compte de ce qui est arrivé; derrière lui, la guitare fait encore quelques pirouettes : ils lui ont volé son tempo, son espace, sa chanson! Django signe d'un clin d'œil en citant Gershwin.

Après cet exploit, les collaborations de Django Reinhardt aux séances d'enregistrement de chanteurs et chanteuses français s'espaceront, puis cesseront. Les essais effectués avec Jacotte Perrier, en 1938, et Josette Daydé, en 1940 - deux femmes enfants (Beryl Davis sera, pour deux titres, leur alter ego britannique) -, laisseront trois aimables comptines 
(Ric et Pussy, Les Salades de l'oncle François et Cou-cou), mais seront sans doute jugés peu convaincants par les membres du Quintette puisqu'ils resteront sans lendemain. L'hommage rendu à Jean de La Fontaine (La Cigale et la Fourmi) en compagnie de Charles Trenet, le 12 mars 1941, apparaîtra sympathique mais anecdotique. C'est qu'à partir de 1935, Django s'est lancé dans la grande aventure du jazz en commençant à graver, en compagnie de Stéphane Grappelli et de leurs complices rythmiciens, une série de titres, originaux et standards, tous plus riches d'invention les uns que les autres. Parmi ces faces, six se feront avec Freddy Taylor, chanteur, trompettiste, danseur de claquettes et apparemment musicien rompu à toutes les ficelles du swing, sur la tête duquel nous poserons finalement la couronne (éphémère: deux séances en mai et octobre 1936) de "chanteur du Quintette du Hot Club de France». Mais c'est une autre histoire...

Une chose étonne si l'on se retourne sur ces quatre années passées en compagnie de vedettes de la chanson française : les collaborations avec ces chanteurs et chanteuses ne suivent pas le mouvement général de l'œuvre de Django qui l'emporte dans le jazz. Il y a en effet plus de jazz avec Éliane de Creus et Jean Sablon en 1933 qu'avec Jean Tranchant, Yvonne Louis et Nane Cholet en 1936. À partir d'avril 1935 (Blue Drag marque un jalon), et à l'exception de la séance du 7 décembre de la même année où sont gravés le Cette chanson est pour vous, Madame auquel nous avons fait un sort et Rendez-vous sous la pluie (une introduction d'une puissance poétique égale à celle de Je sais que vous êtes jolie), les solos les plus intrépides et les plus réussis du guitariste sont ceux qu'il prend au sein $\mathrm{du}$ Quintette à cordes. Comme si après avoir entrevu une possible alliance entre jazz et chansonnette, le résultat à la fin ne pouvait être que la séparation. C'est que le jazz est exigeant: il absorbe toute l'énergie du guitariste (se retrouver sur la même ligne que Coleman Hawkins n'est pas une expérience qui laisse indemne). C'est aussi qu’il est généreux : il offre à Django les espaces les plus propices à l'épanouissement de son génie. Et qu'il est substantiel : Django n'a plus besoin de rien d'autre. Les années chanson française, notamment le brin de route effectué aux côtés de Jean Sablon, resteront celles d'une révélation - révélation de cet exceptionnel inventeur de musique au public, mais aussi, et peut-être d'abord, à luimême. Dans les espaces limités que lui laisse l'accompagnement des chanteurs, le guitariste prend conscience de ses pouvoirs : ils sont sans limites. 


\section{Une empreinte pour la postérité}

Dans son ouvrage Chansons. La bande-son de notre histoire, Louis-Jean Calvet écrit, à propos de la « chanson française du milieu des années 1930 »:

«[...] [L]es instruments devaient s'effacer devant la voix. En écoutant les disques de l'époque, on perçoit un va-et-vient systématique entre la musique et le texte, entre la voix de l'interprète et l'orchestre, qui joue en sourdine quand l'artiste chante et monte en puissance entre les couplets et les refrains. Il y a dans ce partage de l'espace sonore une sorte de signature de l'époque, comme une esthétique, qui n'est pourtant que le produit des conditions techniques d'enregistrement " (2013: 94).

En considérant l'échantillon de la "chanson française des années 1930 » qu'offrent les collaborations de Django Reinhardt, il apparaît que deux écoles se distinguent. D'un côté la "vieille » : l'héritage du "Caf'-conc"; d'un autre la «nouvelle » : l'ouverture à l'influence de la variété américaine et du jazz. Pour la "vieille école»: Jean Tranchant, un des rares sinon le seul auteur-compositeur-interprète de cette troupe, Nane Cholet, Aimé Simon-Girard, Léon Monosson, Germaine Sablon, Yvonne Louis... Plaçons Pierre Lord, chanteur-fiction ${ }^{3}$, entre deux eaux. Pour la «nouvelle école " : Jean Sablon, tout seul, et seulement interprète. Mais empressonsnous de lui adjoindre ses accompagnateurs, qui représentent la première génération des jazzmen français, et ses auteurs et compositeurs, dont certains seront à l'occasion également interprètes : Mireille et Jean Nohain, Bruno Coquatrix, Henri Christiné, Alec Siniavine, Michel Emer, Charles Trenet et Johnny Hess. N'oublions pas les frères Jean et Pascal Bastia, auteurs-compositeurs de l'opérette Dix-Neuf Ans qui nous a laissé le duo fugace Parce que je vous aime, avec la juvénile Éliane de Creus, première association enregistrée entre Sablon et Django. Le duo Pills et Tabet commet également des opérettes où l'ombre du jazz se profile parfois. Louis Palex et Louis Hennevé se sont faits, tantôt ensemble tantôt séparément, une spécialité de l'adaptation des succès américains.

D'ailleurs, la distinction chanson française vs chanson américaine ne recoupe pas mécaniquement la distinction "vieille école» vs «nouvelle école "; Germaine Sablon interprétant Un jour sur la mer (I Cover The

3. Daniel Nevers (cf. le livret de l'Intégrale Django Reinhardt, 3. Djangology (1935), Vincennes, Frémeaux \& Associés, 1996, non paginé) nous apprend que "Pierre Lord " est le nom générique du chanteur de charme de la firme Ultraphone. Sous ce même et unique pseudonyme, plusieurs individus ont officié. Django Reinhardt n'accompagne Pierre Lord que pour une seule séance d'enregistrement, il ne nous est donc pas possible de juger différentes incarnations du charmeur de chez Ultraphone. 
Waterfront) et J'suis pas un ange (I'm No Angel) ne laisse aucun doute sur ce point. Pas plus qu'elle ne signale une absence de recherche et d'innovation du côté des anciens, comme pourrait le laisser supposer l'analyse proposée par Louis-Jean Calvet. Un exemple avec deux ouvres, chacune illustrant l'une et l'autre école, qui constituent deux réussites dans le rapport qui s'établit entre texte et musique, toutes deux novatrices dans le choix des instruments qui accompagnent et toutes deux enregistrées en 1935 : Chanson tendre, de Jacques Larmanjat et Francis Carco, dans l'interprétation de Fréhel; Rendez-vous sous la pluie, de Charles Trenet et Johnny Hess, dans l'interprétation de Jean Sablon. Il semble que la différence entre "vieux style» et "nouveau style» tienne avant tout, pour les vocalistes, à la diction et à l'usage des effets dramatiques; pour les instrumentistes et l'orchestration, à la présence ou non de la syncope jazzée.

C'est là qu'intervient la question du micro. Dans les histoires de la chanson française, le nom de Jean Sablon apparaît toujours associé à l'apparition du microphone. Pour certains auteurs, il s'agit là d'une révolution: "Dans la chanson, il y a un avant et un après Jean Sablon ", affirme Bertrand Dicale (2011 : 77). Et il situe l'événement fondateur au théâtre Mogador, en 1936, où le premier crooner français présente un récital en chantant tout au long derrière un micro - ce qui lui vaudra d'être surnommé "Jean-sans-son". Mais, dès mars 1933, au Théâtre Daunou, Jean Sablon s'était présenté sur scène avec un micro et avec ses accompagnateurs. Pour d'autres, le bouleversement interviendra deux ans plus tard. Lucien Rioux, cité par Louis-Jean Calvet, " considère que la chanson moderne est née en 1938, lorsqu'un jeune homme blond aux cheveux fous" (on a reconnu Charles Trenet, le "fou chantant») créa, à l'ABC, Je chante (in Calvet 2013: 95). En fait, le microphone était utilisé pour les séances d'enregistrement depuis le milieu des années 1920 (Malson \& Tornior 1997). Et réduire la nouveauté apportée par Jean Sablon à un aspect technique - l'amplification de la voix - ne lui rend pas justice: le chanteur aperçoit tout le parti esthétique qu'il peut tirer de l'outil mis à sa disposition et il travaille l'accord entre l'amplification, le phrasé et le timbre de la voix. Du même coup, il modifie la composition de l'orchestre qui l'accompagne: un piano seul ou un piano avec une ou deux guitares et une contrebasse, auxquels il ajoute parfois une clarinette ou un sax alto, les deux instruments tenus par André Ékyan. La relation entre vocaliste et instruments telle que la décrit Louis-Jean Calvet dans la phrase citée en ouverture, l'alternance des parties chantées et des parties orchestrales, demeure, mais en plus, les musiciens dialoguent sans la couvrir avec la voix ou brodent un commentaire tout autour d'elle. L'opposition entre Jean Tranchant et Jean Sablon peut faire là figure de 
cas d'école. Tout autant que Sablon, Jean Tranchant admirait Django et, tout comme lui, il invitera le tout jeune Quintette du Hot Club de France à se produire sur scène à ses côtés, non pas dans l'intimité d'un petit théâtre comme le Daunou, mais dans la prestigieuse salle Pleyel. Si l'on se fie aux enregistrements, incontestablement le guitariste prend ses aises en compagnie de Sablon alors qu'il reste des plus discrets, voire carrément absent $^{4}$, avec Tranchant.

\section{Succès, postérité}

La soirée organisée par Jean Tranchant à Pleyel ne laissa pas une grosse impression au public qui y assista. Les petits ensembles jazz réunis par Jean Sablon ne récoltèrent pas le succès escompté et c'est sur le coup de la déception que le chanteur choisit de s'exiler aux États-Unis et en Amérique du Sud, où il connut la célébrité en cultivant le personnage $\mathrm{du}$ "French Lover». Les enregistrements effectués par Django Reinhardt en compagnie de chanteurs et chanteuses pour les firmes Ultraphone, Gramophone et Polydor ne réalisèrent, signale Daniel Nevers, que des "ventes faibles». Les historiens de la chanson française dont j'ai consulté les ouvrages ne mentionnent même pas ces séances - à peine Louis-Jean Calvet évoque-t-il "l'immense guitariste de jazz Django Reinhardt " qui "accompagnait" Jean Sablon (2013 : 95). Plutôt que Je sais que vous êtes jolie, Cette chanson est pour vous, Madame et Rendez-vous sous la pluie que nous présentons comme des chefs-d'œuvre, ils font un sort à Vous qui passez sans me voir et Je tire ma révérence, enregistrées sans Django, qui furent saluées par la faveur du grand public. Les œuvres qui aujourd'hui provoquent notre admiration sont, au moment de leur création, quasiment passées inaperçues. Est-il possible d'entendre ces chansons enregistrées dans les années 1930 comme les entendaient les contemporains?

Ces auditeurs étaient-ils frappés du même étonnement qui nous saisit à chaque apparition de la guitare de Django Reinhardt ? Notre appréciation des chansons des années 1930 où Django est présent peut-elle s'affranchir de ce que nous connaissons de lui, à la même période, dans les années qui vont suivre, ce jusqu'à sa mort en 1953, et même du destin posthume de sa musique? Les vocalistes avec lesquels Django enregistre appartiennent aussi bien à la "vieille école " qu'à la "nouvelle"; aujourd'hui tous sonnent datés, même Jean Sablon, dans le lot le seul représentant exclusif de la "nouvelle école», et les autres qui apparaissent désuets, certains ringards. La guitare échappe à tout cela. Elle ne sonne ni «ringarde »

4. Daniel Nevers signale (cf. le livret de l'Intégrale Django Reinhardt, 3. Djangology (1935), op. cit.) qu'il arrivait à Django de quitter le studio avant la fin de la séance quand la musique qu'on enregistrait l'ennuyait. 
ni «désuète» ni «datée »; elle sonne «Django ». Django Reinhardt est de ces musiciens de jazz que l'on reconnaît dès les deux premières mesures ou le premier accord. En étant juste lui-même, Django traverse les écoles, les styles, les époques.

Mais "sonner daté ", qu'est-ce que cela veut dire ? Les chansons possèdent, selon l'heureuse expression de Stéphane Hirshi, «l'art de fixer l'air du temps» (2008). C'est-à-dire la faculté de conserver et de restituer ce qui définit de la manière la plus singulière une époque ou un milieu. Ce faisant, la chanson marque son appartenance à cette époque et à ce milieu $^{5}$. Il semble que la guitare de Django Reinhardt échappe à cette dimension : elle est une voix - qui certes évoluera au cours des vingt années que durera la carrière du virtuose, mais restera tout au long immédiatement identifiable - et cette voix se dérobe à toute assignation, elle ne réfere qu’à elle-même. L'intensité de sa présence est telle qu'elle oblitère toute appartenance à une actualité. Inévitablement, cette façon de voir nous conduit à séparer Django soliste de Django au milieu de ses pairs. Jean Sablon, malgré son tact et son élégance, sonne "années 1930 ", le Quintette à cordes, malgré l'originalité de sa formule, la perfection et la chaleur de ses exécutions sonne " années 1930 », pas la guitare soliste - qu'elle expose un thème, façonne un chorus improvisé, ponctue d'un break ou déroule un contrechant autour de la voix d'un chanteur. C'est la raison pour laquelle toute intervention de Django Reinhardt est entendue comme une apparition. Ici. Maintenant.

S'il n'a pas été salué par le succès auprès du public contemporain, le flirt entre la grande figure du jazz à venir et la chanson française de variétés n'est pas resté sans postérité. C'est la part « historique » de l'art de Django, celle qui sonne "datée" et appartient à l'époque, qui féconde cette postérité, et non la part "voix unique qui impose sa fulgurance». Une des originalités de la déclinaison du jazz spécifiquement française élaborée tout au long des années 1930 est la présence d'une guitare au sein des différentes formations, quelle que soit leur taille, du trio au grand orchestre. Or, ces guitares participent de ce que l'on a appelé l'«école Django" ou l' "école gitane ${ }^{6}$ de la guitare jazz. On les retrouve, ces guitares à la couleur bien spécifique, dans les orchestres de Michel

5. La chanson comme marqueur d'époque, les réalisateurs de fictions filmées usent et abusent de ce procédé. Il suffit d'une chanson en fond sonore de n'importe quelle scène de rue ou d'intérieur pour que celle-ci, avant même l'apparition des personnages, de leurs costumes et des décors, soit historiquement située.

6. Je ne reviens pas sur ce débat déjà fait: cf. «Un héritage sans transmission : le jazz manouche », in Jean Jamin \& Patrick Williams (2010 : en particulier 231-233) ; voir aussi Patrick Williams (2000). L'appellation «guitare manouche » viendra plus tard. 
Warlop, d'Alix Combelle, de Patrick et, plus proche de la variété, chez Grégor et ses Grégoriens, Ray Ventura et ses Collégiens, et, plus tard, Jacques Hélian, Wal-Berg, entre les mains du maître Django ou de musiciens, gitans ou pas, qui ont grandi, même lorsqu'ils étaient plus âgés que lui, sous son aile. Les plus grandes réussites swing de Charles Trenet (Terre!, Un rien me fait chanter, Verlaine, etc.) ont été réalisées avec Le Jazz de Paris, dirigé par le saxophoniste Alix Combelle, au sein duquel la guitare était tenue par Joseph "Nin-Nin " Reinhardt, le frère cadet de Django. Et, entre 1937 et 1942, dans Je chante, c'est Jean «Cerani » Maille qui officie, Roger Chaput dans Vous oubliez votre cheval, Lucien Gallopain dans Swing Troubadour, et Eugène "Ninine " Vées, le cousin germain dans Le Temps des cerises... Une ou deux décennies plus tard, nous trouverons Henri Crolla aux côtés d'Yves Montand, d'Édith Piaf, Jean "Matelot » Ferret, de Mouloudji...

Puisque nous en sommes à évoquer le mariage entre le jazz et la musique populaire française, n'oublions pas un courant - que Django Reinhardt n'a fait qu'effleurer - dont le développement peut apparaître paradoxal. Il s'agit de ce que l'on appelle le swing-musette, qui, principalement de 1935 à 1945, connaît un grand succès. Pourquoi " paradoxal » ? Parce que la présence ou l'absence de l'accordéon dans l'accompagnement d'une chanteuse ou d'un chanteur paraissait un critère sûr pour faire le départ entre "vieille école " et "nouvelle école». Or le swing-musette est basé sur l'alliance entre un accordéon, qui ne renie rien de ses origines musette, et une ou deux guitares, de celles qui ressortissent au «style gitan» (et dont les représentants les plus brillants, les frères Ferret - Sarane, Baro, Matelot - et Didi Duprat, seront là aussi, gitans ou pas), qui assurent une rythmique de type jazz et se partagent les chorus sur un répertoire adapté ou inspiré de la variété américaine.

\section{Événement, monument}

Publie-t-on aujourd'hui Aimé Simon-Girard, Léon Monosson, Nane Cholet, Nita Rette, Yvonne Louis... ailleurs que dans une Intégrale Django Reinhardt? Les chansons qu'ont gravées ces artistes se trouvent réduites à n'être plus que des témoins de leur époque - rôle qui se révèle puis s'impose au fil du temps. Une réédition leur redonne-t-elle vie? Plutôt, elle les fige dans leur statut de pièces de collection, vestiges de peu de prestige ou faciles déclencheurs de nostalgie. C'est le sort qui les attend, sinon l'oubli. À moins que... Plus de soixante-dix ans après l'enregistrement réalisé par Mireille et Jean Sablon, Françoise Hardy et Jacques Dutronc reprennent, sans ironie, sans nostalgie, Puisque vous partez en voyage: la pièce de musée redevient une œuvre vivante. Les reprises remettent dans 
le mouvement de la vie quand les rééditions pétrifient? Je ne vais pas ici reprendre la démonstration faite à propos des standards dans le jazz ${ }^{7}$. Mais avec Django, les choses ne se passent pas ainsi. Les rééditions confirment la force de l'élan vital que l'enregistrement a capté, qu'il rend répétable à volonté et qu'il est impuissant à momifier ; les reprises entrent en compétition avec les rééditions - et c'est toujours Django qui gagne! À peine servent-elles à mesurer la distance qui sépare le modèle de ses suiveurs.

Ce qui nous touche n'est-ce pas le sentiment d'être associé à un moment unique? - sentiment que la répétition sur commande ne réussit pas à réduire. Il est vrai que nous sommes émus davantage quand telle chanson, telle improvisation que nous aimons retentit à un moment ou dans un cadre où nous ne l'attendons pas. L'arrivée de la guitare de Django Reinhardt dans l'univers des chanteurs de charme et des chanteuses réalistes à tous les coups saisit, inattendue: "apparition » est le mot que nous avons employé pour en rendre compte.

Donc une chanson, près d'un siècle après son enregistrement, peut être à la fois un témoin historique, une pièce de musée, un moment d'art vivant. C'est bien ce que montrent les fragiles monuments édifiés par Django Reinhardt et les chanteurs et chanteuses de variétés au milieu des années 1930.

Bien sûr, on fera remarquer qu'une telle appréciation tient à l'admiration que j'éprouve pour la musique de Django Reinhardt... Bien sûr. Mais les auditeurs qui ne connaissent pas ces œuvres adoptent la même attitude lorsqu'ils les découvrent. C'est ce qu'il s'est passé lors de l'exposé au séminaire "Chansons", le 3 avril 2012. La partie du public qui ne connaissait pas ces enregistrements a partagé mon enthousiasme (il est vrai que, soucieux d'élever le niveau de l'exposé, j'avais multiplié l'audition d'extraits musicaux), ravie dans le présent qu'installe la guitare et comprenant que celui-ci participait d'une exigence de méthode, seule attitude permettant de capter les qualités de la musique. En effet, comment en saisir à la fois la force et les subtilités si l'on n'est pas animé par la passion?

L'analyse musicologique que propose André Hodeir du solo de Django dans Solid Old Man, enregistré le 5 avril 1939, en compagnie de trois musiciens de l'orchestre de Duke Ellington alors de passage à Paris (Rex Stewart, Barney Bigard et Billy Taylor), si elle met bien en évidence l'inventivité de l'improvisateur et son art d'éviter truismes et facilités, ne dit cependant rien de l'essentiel: la qualité unique de cette voix (Hodeir 1984 : 149-150).

7. Cf. le chapitre «Standards et standardisation: sur un aspect du répertoire des musiciens de jazz», in Jean Jamin \& Patrick Williams (2010 : 147-202); voir aussi Patrick Williams (2006b). 
Un même commentaire, émanant principalement des spécialistes du jazz, accompagne la présentation de Django Reinhardt dans les dictionnaires et les encyclopédies : il y a dans sa musique "quelque chose qui vient d'ailleurs". Ce "quelque chose», Frank Ténot le définit sans ambages: "La musique de Django est le résultat de la rencontre de l'héritage tsigane et du jazz des années 1930: folklores nés dans des ghettos en marge des cultures officielles» (1988: 851-852).

"L'héritage tsigane": voici que se profile la redoutable question du caractère ethnique de la musique créée par Django Reinhardt. Cette question, nous avons eu à la traiter dans tous les travaux que nous avons consacrés au guitariste manouche. Tant qu’à répéter des considérations déjà proposées, il m'a semblé préférable, même si l'autocitation place toujours dans une position inconfortable, de citer ces travaux plutôt que de les paraphraser. Pour les membres de la famille Reinhardt, au début du XXe siècle, la musique est un métier parmi d'autres :

«Il ne semble pas qu'il existe à cette époque une tradition musicale spécifique aux Manouches [...]. Aucun témoignage littéraire, aucun récit de voyageur n'évoque une telle tradition, comme c'est le cas pour les Gitans d'Espagne ou pour les Roms de Hongrie. Il s'agit de gagner sa vie, donc avant tout de faire plaisir au public. Jouer de la musique dans de telles conditions favorise l'éclectisme : il faut savoir répondre à la demande, et l'exhibition de virtuosité : il faut impressionner l'assistance. Quelle musique pouvait séduire les habitants de Liberchies ${ }^{8}$ aussi bien que ceux de Nice ou de Toulon ${ }^{9}$. Adaptations d'ouvertures d'opéras célèbres et airs d'opérettes, succès du Caf'-conc et du cabaret, probablement des mélodies qui, au fil des années, avaient fini par être connues par toutes les couches de la population et dans toutes les régions: un répertoire populaire qui n'était pas un folklore [...]. Si cette musique ne révèle pas une tradition singulière, elle possède cependant une spécificité qui tient à l'instrumentation: la formation n'est composée que d'instruments à cordes [...]. Faut-il voir dans cette instrumentation tout-cordes une fidélité ethnique? Que ce soit en Europe orientale (le violon, le cymbalum) ou en Espagne (la guitare), la virtuosité tsigane s'exerce de manière privilégiée sur des instruments à cordes - pincées, frottées, percutées. Ou bien faut-il y voir une nécessité pratique? Ces instruments sont les compagnons des musiciens qui courent les routes. Ou bien encore un choix esthétique? Indéniablement, l'alliance des cordes frottées et des cordes pincées donne une couleur singulière à la musique. Ou faut-il considérer les trois options à la fois? Les Tsiganes ont appris à tirer un parti pris esthétique de contraintes pratiques; c'est peut-être même, à leurs yeux, ce qui constitue leur talent particulier; ainsi la fidélité à de telles formules leur apparaît-elle comme une fidélité à eux-mêmes " ${ }^{10}$.

8. Le village du Hainaut, en Belgique, où Django est né.

9. Villes que la famille Reinhardt-Weiss a régulièrement fréquentées durant l'enfance de Django. 10. Cf. le chapitre "Un héritage sans transmission : le jazz manouche», in Jean Jamin \& Patrick Williams (2010 : 225-251); voir aussi Patrick Williams (2000). 
La collaboration avec les artistes de variétés pourrait-elle servir de pierre de touche? Révéler, hors de tout contexte "traditionnel ", la part ethnique qui tout au fond anime la création de Django?

Je n'entends rien de cela, rien qui renvoie à un "héritage ethnique", quelle que soit la communauté à laquelle on puisse se référer, rien à un «folklore», pour reprendre les mots de Frank Ténot. Je n'entends que la part de génie singulier qu'apporte Django. S'il existe un accent tsigane, un accent manouche, dans ce que joue Django Reinhardt au milieu des années 1930, c'est lui qui l'invente.

Le «jazz manouche ", c'est-à-dire la pléthore d'imitateurs de Django Reinhardt, principalement du Django des années 1934-1945, chargera la voix singulière de cette guitare d'une couleur bohémienne. Mais ce mouvement apparaîtra sur la scène publique et se développera à partir des années 1960 ; il ne deviendra l'emblème des Manouches, même quand ses interprètes n'appartiendront pas à ce groupe, parmi les Tsiganes, que progressivement. La "pompe", cette manière si caractéristique pour les guitares d'assurer le soutien rythmique en marquant d'un accord chaque temps de la mesure, véritable signature de l'appartenance manouche, n'a pas toujours été perçue ainsi. Paul-Louis Rossi, chroniquant en 1964 une réédition de Django avec le Quintette tout-cordes, évoque, à propos de "cette manière de moudre le temps": "une "élégance toute française " ${ }^{11}$. Méfions-nous des écoutes rétrospectives - mais comment faire, avions-nous déjà remarqué, pour ne pas entendre la musique d'hier avec les oreilles d'aujourd'hui ? Confiance à Django, à la force de sa parole qui se joue, avions-nous aussi affirmé, de toute appartenance à une quelconque actualité.

Si nous remplaçons l'" héritage ethnique " par l'« invention de Django", peut-être convient-il alors de ne plus chercher la dimension ethnique dans le contenu mais dans une attitude? La dimension sociologique n'est pas absente de la définition de Frank Ténot lorsqu'il évoque les "ghettos en marge des cultures officielles ». Les Tsiganes ont en effet la réputation de faire leur vie dans les marges de la société. D'échapper à la règle citoyenne en se glissant dans les failles de la loi. D'ignorer la norme. D'édifier des espaces de vie dans des lieux qui ne sont pas destinés à cela, "passeurs de murailles, transgresseurs de frontières ", comme l'écrit Chris Flicker dans le texte de la pochette de l'album enregistré en duo par le contrebassiste Charlie Haden et le guitariste Christian Escoudé, intitulé Gitane, publié en $1979^{12}$. Dans notre examen des chansons des années 1930,

11. In Jazz Magazine, 1964, $110: 47$.

12. Cf. Charlie Haden \& Christian Escoudé, Gitane, All Life - AL 001, 1979. 
nous avons insisté sur la faculté que possède la guitare de Django de se glisser dans les interstices, de s'emparer des espaces qui ne lui étaient pas dévolus... Un tel portrait participe autant de l'imagerie que du réel, de la fantasmagorie que de la sociologie. Mais est-il possible de dresser un portrait des Fils $d u$ vent qui trouve quelque écho auprès du public sans faire appel aux clichés? Django Reinhardt ne peut être qu'un Gitanhéritier-d'une-tradition, un Gitan-porteur-d'un-mystérieux-atavisme, quand bien même sa discographie des années 1930-1936, pour autant qu'une discographie rende compte d'un parcours musical, révèle une diversité d'expériences qui montre avec éclat son appartenance à la scène parisienne du tout jeune «jazz à la française». Encore faudrait-il hiérarchiser ces expériences - une séance avec Nane Cholet ou Léon Monosson vaut-elle une séance avec Coleman Hawkins et Benny Carter, ou avec les complices du Quintette du Hot Club de France? - et ne pas oublier que s'il y a dans l'art de Django une part "voix unique" sur laquelle le temps n'a pas de prise, il y a aussi une part "historique».

À ce moment de son évolution, ce que Django apporte dans les chansons, n'est-ce pas ce que le jazz lui apporte? C'est-à-dire des espaces pour déployer son imagination, un balancement rythmique dont le soutien jamais ne fait défaut, un traitement du son qui offre à tout musicien de cultiver sa voix propre ?... Il n'y aurait alors, pour le Django de la chanson française comme pour le Django de toute son œuvre, que la rencontre avec le jazz.

Sous la gouverne ou hors de la prégnance des images et des stéréotypes, cette question du rapport entre l'appartenance manouche et la singularité du jeu de Django Reinhardt ouvre sur un problème plus vaste. Est-il possible, est-il pertinent d'établir un lien entre un style de vie et un style musical ? N'abordons-nous pas là le domaine de l'ethnomusicologie? Cette discipline a-t-elle apporté une réponse à cette interrogation? Ou bien autant de réponses qu'elle a étudié de cas d'espèces? Que dit-elle à propos des musiques populaires saisies par l'industrie du spectacle?

Formes brèves, pouvoirs sans limites

1933-1936: • la chanson de variétés française balance entre drames réalistes et roucoulades sentimentales d'un côté, élans swing et poésie teintée de fantaisie de l'autre, elle semble devoir bientôt basculer mais en fait, "vieille « école » et " nouvelle école » continueront à cohabiter, parfois dans le répertoire d'un même interprète, celui d'Édith Piaf en donne un bon exemple ;

- Django Reinhardt n'est encore qu'un virtuose sans formation savante en quête d'un langage, il vient de le trouver. 
Les deux trajectoires, celle d'un domaine d'expression populaire et celle d'un créateur d'exception, se croisent. Mais les incursions de Django dans le monde de la chanson paraissent accessoires à côté de son engagement dans le jazz. Pour le guitariste sans bagage, les enregistrements avec les chanteurs et les chanteuses s'intercalent, nous l'avions remarqué, avec la participation à des sessions de jazz à la française, des collaborations avec des musiciens américains, les premiers pas du Quintette du Hot Club de France tout cordes. L'entrée dans la création et l'entrée dans le métier se confondent. Il faut y ajouter le succès auprès du public qui viendra presque immédiatement pour toutes ces expériences, à l'exception de la chanson. Le Quintette du Hot Club de France deviendra plus célèbre que tous les chanteurs que ses membres ont accompagnés. Django Reinhardt accomplira son œuvre au sein du jazz de son temps :

- Des pièces d'une durée de trois minutes: des thèmes originaux, signés Django Reinhardt ou "Django Reinhardt" avec un complice du Quintette, Stéphane Grappelli, Hubert Rostaing, Gérard Lévêque, Eddie Bernard $^{13}$ (tous n'accoleront pas leur nom à celui de Django, mais leur concours fut bien réel); des chansons de Tin Pan Alley, certaines qui sont des standards, certaines quasi inconnues; des compositions signées par des figures du jazz, Duke Ellington par exemple; des blues improvisés dans le studio au moment d'un enregistrement. Max Robin et Jean-Philippe Watremez ont publié le répertoire des thèmes originaux et des blues (s.d.).

- Des solos de trente-deux, seize, huit mesures, des breaks, des introductions, des ponctuations : des inventions brèves dans des formes brèves.

Django Reinhardt est un maître du chorus. La chanson n'a pas représenté un dépaysement pour lui dans sa marche vers l'élection du jazz. Et probablement n'aurait-il pu multiplier les interventions éblouissantes derrière ou aux côtés des chanteurs et chanteuses s'il ne s'était embarqué dans le jazz en même temps. Mais la structure d'une chanson, avec l'alternance couplet-refrain, n'offre pas les mêmes espaces que le schéma thème-variations-thème cultivé par le jazz classique ${ }^{14}$. Pourtant, tout autant que ses enregistrements de jazz, ceux réalisés avec les vocalistes des années 1930 donnent le sentiment que les pouvoirs de Django ne

13. Quelques auteurs (Jacques Larue pour Swing 39, qui devient Je t'aime, et pour Nuages; Francis Blanche pour Fleur d'ennui; Lawrence Reisner pour Swing 42, qui devient Swing-Rêverie, et pour Mélodie au crépuscule) mettront des paroles sur des mélodies composées par Django Reinhardt. Ces chansons trouveront des interprètes célèbres à l'époque: Lucienne Delyle, Lys Gauty, Rina Ketty, Irène de Trébert, Guy Berry. À l'exception de Mélodie au crépuscule, le 7 juillet 1943 avec un orchestre de gala et la chanteuse moins connue Nelly Kay, Django n'enregistrera pas ces thèmes avec paroles.

14. Pour des précisions sur la manière dont le jazz s’y prend pour transformer les chansons en «thèmes", cf. Philippe Baudoin (2001 [1990]). 
connaissent pas de limites - c'est sur ce point que j'avais choisi de conclure mon article et mon exposé.

Les pouvoirs de Django ? Le pouvoir d'inventer des histoires, le pouvoir de conduire un récit, le pouvoir d'allier surprise et cohérence, le pouvoir de transfigurer le matériau, le pouvoir de faire sien un idiome venu d'ailleurs, le pouvoir d'étonner le public, ses pairs musiciens, lui-même...

Mais n'est-ce pas le fait qu'il existe des limites qui donne l'impression de pouvoirs illimités? Les limites, Django les fait apparaître parce qu'il s'en échappe. Son génie tout entier dans cette échappée? Alors Daniel Nevers a raison quand il proclame: «Si Django n'avait enregistré que ces quelques mesures, on saurait quand même quel immense musicien il fut ${ }^{15}$.

L'œuvre de Django Reinhardt n'est-elle qu'une addition d'instants précieux? Mais qu'est le jazz?

Certains biographes de Django Reinhardt (Bessières 2012 : 135 ; Robin 2010 : 19) rapportent qu'à un moment de son évolution, s'attelant à la composition d'une symphonie, il aurait déclaré: "J'entends des choses que je ne peux jouer» - Stéphane Grappelli renchérit : "Je crois qu’il entendait plus de musique qu'un orchestre complet était capable d'en jouer" (cité in Bessières 2012 : 128). On imagine l'étendue de la frustration qui se cache derrière une telle confidence. Songeait-il à son défaut de formation académique, aux limites de la guitare, au carcan des formes brèves? Nous connaissons les modalités selon lesquelles il composait. Un compagnon note sur le papier ce que Django invente sur sa guitare - mélodie et harmonie. Le point final se règle au moment de la performance, entre musiciens, comme cela est courant dans le jazz. Quand il faut produire une orchestration pour une formation plus étoffée que le Quintette, Django joue la partie de chaque section ou pupitre sur la guitare et le partenaire porte-plume transcrit. À plusieurs reprises au cours de sa carrière, il a cédé à la tentation de la "grande forme ". Il a ébauché une symphonie, composé une Messe pour orgue, il a conçu plusieurs projets de musiques pour des ballets en collaboration avec des danseurs et chorégraphes aussi réputés qu'Yvette Chauviré ou Serge Lifar, il a entrepris, avec André Hodeir ${ }^{16}$, une musique pour un film que devait

15. Cf. Daniel Nevers, livret du coffret de l'Intégrale Django Reinhardt, 1. Présentation stomp (1928-1934), op. cit.

16. André Hodeir, violoniste, compositeur, musicologue et critique de grande autorité, auquel nous empruntons la distinction entre "petite forme " (nous avons préféré "forme brève») et "grande forme" (cf. Hodeir 1981 [1954]). Ce dernier estime que "Django est un musicien qui n'est pas allé au bout de ses dons: ses virtualités restent à mon avis plus grandes que son œuvre même " (in Jazz Hot, 1963, 187 : 28). 
tourner Marcel Carné. Mais la partition de la symphonie s'est perdue - il ne reste que le thème intitulé Manoir de mes rêves (Django en a enregistré neuf versions différentes) ${ }^{17}$; de l'enregistrement de la Messe, jouée en 1944 par Léo Chauliac à la Chapelle de l'Institut des jeunes aveugles, à Paris ${ }^{18}$, seul a été conservé un vestige d'une durée de sept minutes et demie ; les musiques pour les ballets et pour le film n'ont jamais vu le jour... Et, bien que les quelques minutes de la Messe impressionnent, on ne peut savoir quel intérêt présentaient ou auraient présenté ces entreprises. Django Reinhardt restera cet enchanteur qui, dans le cadre que lui offrent thèmes de jazz et chansons, nous éblouit par ses fulgurances et ses éclats de funambule. S'essayant à la "grande forme", l'aurait-il conservé, cette qualité de funambule?

Centre national de la recherche scientifique, Paris

MOTS CLÉS/KEYWORDS : Django Reinhardt (1910-1953) - chanson française (histoire)/French chanson (history) - chanson populaire (sociologie)/popular song (sociology) - interprétation/ performance - jazz.

17. Cf. l'interview de Jean-Marie Pallen, «Django et le projet de symphonie », in Guitarist Acoustic Magazine, 2010, numéro hors-série : Django 1910-2010, 2010 : 18-22.

18. Cf. Gypsy Jazz School, Django Legacy, Iris Music - Harmonia Mundi, 2002 [coffret de deux CD]. 


\section{DJANGO REINHARDT ET LES CHANTEURS ET CHANTEUSES DE VARIÉTÉS}

1933-1936

1933

Éliane de Creus \& l'Orchestre du théâtre Danou (Gramophone)

II n'y en a pas deux comme moi

Éliane de Creus \& Jean Sablon (Gramophone)

Parce que je vous aime; Si j'aime Suzy

Jean Sablon (Columbia)

Le Même coup; Je suis Sex-Appeal

1934

Jean Sablon, avec André Ékyan \& son Orchestre Jazz (Columbia)

Le jour où je te vis; Prenez garde au grand méchant loup; Pas sur la bouche

Germaine Sablon, avec Michel Warlop \& son Orchestre (Gramophone)

Un jour, sur la mer; Ici l'on pêche; Toboggan; Celle qui est perdue

J'suis pas un ange ; La Chanson du large

Tendresse; j'ai besoin de toi

Deux Cigarettes dans l'ombre; Je voudrais vivre

Jean Sablon (Columbia)

Je sais que vous êtes jolie; Par correspondance

Aimé Simon-Girard, avec Michel Warlop \& son Orchestre (Gramophone)

Cocktails pour deux; L'Amour en fleurs

1935

Jean Sablon (Columbia)

La Dernière bergère; The Continental; Un baiser

Le Petit Mirsha, avec Michel Warlop et son Orchestre (Gramophone)

Vieni, Vieni ; Maman, ne vends pas la maison! ; Petit homme, c'est l'heure de faire dodo

Léon Monosson (Columbia)

Deux Cigarettes dans l'ombre; Tout le jour, toute la nuit

Pierre Lord (Ultraphone)

Simplement; Fumée aux yeux; Cocktails pour deux

Germaine Sablon \& Jean Sablon (Gramophone)

Un amour comme le nôtre; La Petite île

Nane Cholet (Ultraphone)

Si j'avais été ; Fièvre ; Ainsi soit-il ; Les Quatre farceurs

Nitta Rette \& son Hot Trio (Polydor)

Points roses; Un instant d'infini ; Mon cour reste auprès de toi 
André Pasdoc, avec Louis Vola \& son Orchestre (Polydor)

124 Pourquoi, pourquoi?; Vivre pour toi

Yvonne Louis (Polydor)

Mirages

Jean Sablon (Columbia)

Cette chanson est pour vous, Madame ; Darling, je vous aime beaucoup

Jean Sablon, avec accompagnement d'orchestre (Columbia)

Cette chanson est pour vous, Madame; Rendez-vous sous la pluie

\section{6}

Jean Tranchant, avec Michel Emer \& son Orchestre (Pathé \& Micro Redoute) L'Amour en voyage; Les Baisers prisonniers; Mon bateau est si petit;

Quand il est tard; Le Piano mécanique; Ici l'on pêche

Yvonne Louis (Polydor)

Au grand large

Jean Tranchant (Pathé)

Le roi Marc; Mademoiselle Adeline

Nane Cholet (Pathé)

Terrain à vendre ; Ainsi soit-il 


\section{RÉFÉRENCES CITÉES}

\section{Baudoin, Philippe}

2001 [1990] Jazz mode d'emploi. Petite encyclopédie des données techniques de base, 1 et 2. Paris, Outre Mesure ("Théories»).

Bessières, Vincent, ed.

2012 Django Reinhardt, Swing de Paris. Catalogue de l'exposition, musée de la Musique, 2012-2013. Texte de Michael Dregni.

Paris, Textuel-Cité de la Musique.

Calvet, Louis-Jean

2013 Chansons. La bande-son

de notre histoire. Paris, L'Archipel.

Dicale, Bertrand

2011 Les chansons qui ont tout changé.

Paris, Fayard.

Hirshi, Stéphane

2008 Chanson. L'art de fixer l'air du temps: de Béranger à Mano Solo. Paris, Les Belles

Lettres / Valenciennes, Presses universitaires de Valenciennes ( Cantologie» 6).

\section{Hodeir, André}

1981 [1954] Hommes et problèmes du jazz. Marseille, Parenthèses ("Epistrophy»).

1984 Jazzistiques. Marseille, Parenthèses ("Epistrophy»).

Jamin, Jean \& Patrick Williams

2010 Une anthropologie du jazz.

Paris, CNRS Éd.

Malson, Lucien \& Jean-Claude Tornior

1997 «Abrégé d'histoire de l'enregistrement : [I] - Premières dates ; [II] - Dictionnaire chronologique des techniques ",

Les Cahiers du jazz 10 : 17-32.

Rioux, Lucien

1966 Vingt Ans de chansons en France.

Paris, Arthaud.

Robin, Max

2010 "Django et le projet de symphonie", Guitarist Acoustic Magazine Hors-série : Django 1910-2010: 18-22.
Robin, Max \& Jean-Philippe Watremez

[s.d.] Complete Django. The Ultimate Django's Book. Clamart, Bookmakers International.

Sablon, Jean

1979 De France ou bien d'ailleurs... Paris, Robert Laffont ("Vécu»).

Ténot, Frank

1988 "Reinhardt, Django ", in Philippe

Carles, André Clergeat \& Jean-Louis

Comolli, eds, Dictionnaire du jazz.

Paris, Robert Laffont («Bouquins)».

Williams, Patrick

1998 [1991] Django Reinhardt.

Marseille, Parenthèses ("Eupalinos»). 1999 "Dans le sillon de Django ", Jazzman Hors-série : Jazz et World, la belle histoire : 48-50.

2000 "Un héritage sans transmission: le jazz manouche ", Ethnologie française 30 (3) : 409-422.

2001 "Django, Vous et Moi ", Jazz Magazine 511 : 44-45.

2003 «Django ou la non-disparition", Jazz Magazine 537 : 30-31.

2006a "L'improvisation et le jazz manouche ", in L'Improvisation du jazz. Actes du $2^{e}$ colloque de Monségur. Bordeaux, Presses universitaires de Bordeaux : 29-40.

$2006 b$ "Standards et standardisation: sur un aspect du répertoire des musiciens de jazz ", L'Homme 177-178: Chanter, musiquer, écouter : 7-48.

2007 "Django Reinhard, compositeur", livret de Django Reinhardt Plays Django Reinhardt. Compilation de 5 CD. Arles, Le Chant du Monde : 3-28. 2010 Les Quatre Vies posthumes de Django Reinhardt. Trois fictions et une chronique. Marseille, Parenthèses («Eupalinos»). 2012 "Si bien parti, Django!», Music Magazine. La bande-son des sociétés 3: 58-61. 
Patrick Williams, "Cette chanson est pour vous, Madame...": les années chanson française de Django Reinhardt, 1933-1936. - Entre 1933 et 1935, Django Reinhardt a participé à de nombreuses séances d'enregistrement comme accompagnateur de chanteuses et chanteurs français de variétés. Ces œuvres sont révélatrices à la fois de l'état de la chanson française populaire à cette époque et de l'évolution du guitariste qui deviendra une figure majeure $\mathrm{du}$ jazz. Larticle, dans un premier temps, examine les différentes manières selon lesquelles se règlent les relations entre les vocalistes et Django; dans un deuxième temps, il s'interroge sur ce que peut être l'écoute de ces gravures quatre-vingts ans après leur réalisation.
Patrick Williams, "Cette chanson est pour vous, Madame...": Django Reinhardt's French Popular Music Years, 1933-1936. — From 1933 to 1935, Django Reinhardt took part in many recording sessions as an accompanist for popular French singers. These recordings reveal both the state of popular French music at the time and the evolution of the guitarist, who went on to become a leading jazz artist. The article first examines the different ways in which relations between the vocalists and Django were managed, and then considers how these recordings are likely to be listened to eighty-five years after they were produced. 